\title{
The Visibility Function in Interferometric Aperture Synthesis Radiometry
}

\author{
Ignasi Corbella, Member, IEEE, Núria Duffo, Member, IEEE, Mercè Vall-llossera, Member, IEEE, \\ Adriano Camps, Senior Member, IEEE, and Francesc Torres, Member, IEEE
}

\begin{abstract}
The fundamental equation of interferometric aperture synthesis radiometry is revised to include full antenna pattern characterization and receivers' interaction. It is shown that the cross correlation between the output signals of a pair of receivers is a Fourier-like integral of the difference between the scene brightness temperature and the physical temperature of the receivers. The derivation is performed using a thermodynamic approach to account for the effects of mutual coupling between antenna elements. The analysis assumes that the receivers include ferrite isolators so that the noise wave passing from the receiver toward the antenna can be modeled as uncorrelated ambient noise. The effect of wide beamwidth antennas on the polarization basis of the retrieved brightness temperature is also discussed.
\end{abstract}

Index Terms-Interferometric aperture synthesis, microwave radiometry.

\section{INTRODUCTION}

I NTERFEROMETRIC aperture synthesis was suggested in the 1980s as an alternative to real aperture radiometry for earth observation at low microwave frequencies with high spatial resolution [1]. The first instrument to use this concept was the Electronically Scanned Thinned Array Radiometer, an airborne L-band radiometer using real aperture for across-track direction and interferometric aperture synthesis for along-track [2]. A radiometer using aperture synthesis in both directions [the Microwave Imaging Radiometer Using Aperture Synthesis (MIRAS)] was proposed in [3] to provide soil moisture and ocean surface salinity global coverage measurements from space. The selected configuration was a Y-shape structure having many small receivers evenly distributed along the arms. In May 1999, the European Space Agency (ESA) approved the Soil Moisture and Ocean Salinity (SMOS) mission [4] having MIRAS as the core instrument. Extensive work has already been done to improve the understanding of such a radiometer [5], especially regarding its expected performance [6], calibration [7], and inversion techniques [8]. Airborne instruments using two-dimensional aperture synthesis have been proposed by different laboratories [9], [10] and are at different stages of development. A good review of the recent history of synthetic aperture radiometer systems can be found in [11].

Manuscript received April 11, 2003; revised April 13, 2004. This work was supported by EADS-Casa Espacio and the European Space Agency (ESA) under Contract 15138/01/NL/SF and by the Spanish Administration under Contract TIC2002-04451-C02-01.

The authors are with the Department of Signal Theory and Communications, Universitat Politècnica de Catalunya, 08034 Barcelona, Spain (e-mail: corbella@tsc.upc.es).

Digital Object Identifier 10.1109/TGRS.2004.830641

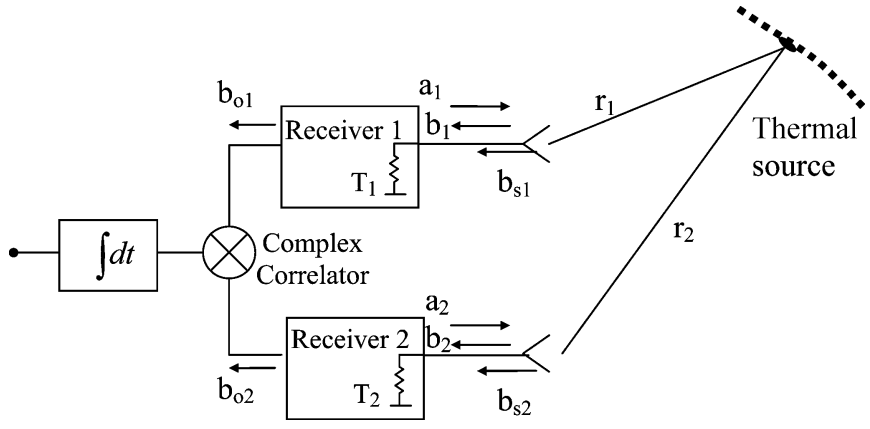

Fig. 1. Pair of antennas pointing to a thermal source.

The fundamental theory behind this technique is the same as the one used for decades in radioastronomy. In summary, the instrument measures the cross correlations between all pairs of receivers to get the so-called visibility function. In a first-order approximation, the brightness temperature of the source is computed as the inverse Fourier transform of this function. However, the large field of view present in earth observation induces nonnegligible effects of individual antenna patterns, obliquity factors, and fringe-washing functions. Recent experimental work [12] has shown that mutual effects of close antennas, as well as their individual matching, become important to fully understand the measurements.

In this paper, a complete formulation of the visibility function, including full antenna characteristics and interactions between receivers, is presented. The main result is that when these effects are taken into account, the measured cross correlation between receiver output signals turns out to be proportional to the inverse transform of the difference between the brightness temperature of the source and the physical temperature of the receivers. This effect, which has been never taken into account in previous approaches, has an important impact on inversion techniques and also on instrument specifications and performance.

\section{FReQuency Domain CorRelation MatriX}

Fig. 1 shows a single baseline of an aperture synthesis radiometer, consisting of a pair of antennas pointing to a thermal source and connected to perfectly matched receivers at physical temperatures $T_{1}$ and $T_{2}$. The system formed by the antennas and the space around them is a linear two-port for which an S-parameter matrix can be defined in the frequency domain. The incident and reflected power waves, denoted by vectors $\mathbf{a}$ and $\mathbf{b}$, respectively, are thus related by the general equation [13], [14]

$$
\mathbf{b}=\mathbf{S a}+\mathbf{b}_{\mathbf{s}}
$$


where $\mathbf{b}_{\mathbf{s}}$ is the vector containing the waves $b_{s_{1}}$ and $b_{s_{2}}$ generated by the thermal source to which the antennas are pointing. The correlation matrix of the total outward waves is readily computed from (1) [15]

$$
\overline{\mathbf{b b}^{\dagger}}=\overline{\mathbf{S a}^{\dagger}} \mathbf{S}+\overline{\mathbf{b}_{\mathbf{s}} \mathbf{b}_{\mathrm{s}}^{\dagger}}
$$

where the dagger indicates Hermitian transpose operation and the overbar the correlation product. This equation can be expanded to ${ }^{1}$

$$
\begin{aligned}
& \overline{\left|b_{i}\right|^{2}}=\left|S_{i i}\right|^{2} \overline{\left|a_{i}\right|^{2}}+\left|S_{i j}\right|^{2} \overline{\left|a_{j}\right|^{2}}+\overline{\left|b_{s_{i}}\right|^{2}} \\
& \overline{b_{i} b_{j}^{*}}=S_{i i} S_{j i}^{*} \overline{\left|a_{i}\right|^{2}}+S_{i j} S_{j j}^{*} \overline{\left|a_{j}\right|^{2}}+\overline{b_{s_{i}} b_{s_{j}}^{*}}
\end{aligned}
$$

where the subscripts $i$ and $j(i \neq j)$ have values 1 or 2 . Since the analysis is performed in the frequency domain, $\overline{\left|b_{i}\right|^{2}}$ represents the power spectral density (total power in a $1-\mathrm{Hz}$ bandwidth) of the noise wave $b_{i}$ and $\overline{b_{i} b_{j}^{*}}$ the cross-power spectral density of $b_{i}$ and $b_{j}$, both having dimensions of watts per hertz.

In general, the thermal noise emitted by the input ports of the receivers of Fig. 1 depends on the specific receiver design [15]. Typically, an isolator is placed at the input of each receiver. In this case, the temperature of the noise traveling from the receiver to the antenna equals the physical temperature of the isolator so that

$$
\overline{\left|a_{i}\right|^{2}}=\mathcal{K} T_{i}
$$

where $\mathcal{K}$ is Boltzmann's constant. The noise waves $a_{i}$ and $a_{j}$ are also uncorrelated in this case. If no isolator is present or has poor isolation, then this partial correlation must be taken into account.

The power and cross-power spectral density of the waves generated by the extended thermal source $b_{s_{i}}$ and $b_{s_{j}}$ are the sum of the contributions coming from all points in space. Assuming lossless antennas

$$
\begin{aligned}
\overline{\left|b_{s_{i}}\right|^{2}} & =\frac{\mathcal{K} D_{i}}{4 \pi} \iint_{4 \pi} T_{\mathrm{B}}(\theta, \phi)\left|F_{n_{i}}(\theta, \phi)\right|^{2} d \Omega=k T_{a_{i}} \\
\overline{b_{s_{i}} b_{s_{j}}^{*}} & =\frac{\mathcal{K} \sqrt{D_{i} D_{j}}}{4 \pi} \iint_{4 \pi} T_{\mathrm{B}}(\theta, \phi) F_{n_{i}}(\theta, \phi) F_{n_{j}}^{*}(\theta, \phi) e^{j k \Delta r} d \Omega \\
& =k \mathcal{V}_{i j}
\end{aligned}
$$

where $d \Omega$ is the solid angle subtended by an elementary source at angular coordinates $(\theta, \phi)$ and $T_{\mathrm{B}}(\theta, \phi)$ the brightness temperature of the source in that direction (see discussion below). The exponential term accounts for the phase difference of the waves coming from a source element to each antenna, so $\Delta r=$ $r_{j}-r_{i}$ and $k$ is the wavenumber $k=2 \pi f / c$ where $f$ is the frequency and $c$ the velocity of light. The term $T_{a_{i}}$ in (6) is the radiometric antenna temperature seen by the receiver in the conditions of Fig. 1 and $\mathcal{V}_{i j}$ in (7) a correlation temperature called visibility due to the similarity of the term used in radioastronomy [16]. The only difference between both is that the radioastronomy visibility is defined as a function of the source

\footnotetext{
${ }^{1}$ Due to the reciprocity theorem, $S_{i j}=S_{j i}$, but this is not a restriction for the present analysis.
}

spectral brightness instead of its brightness temperature, so it has dimensions of flux power spectral density (watter per square meter per hertz) instead of kelvin. The rest of the parameters appearing in (6) and (7) are defined in the following discussion.

The directional characteristics of an antenna are fully characterized by its normalized field pattern $\vec{F}_{n}(\theta, \phi)$, which is a complex vector proportional to the radiated electric field at the given direction. In the far-field region, it has two transversal components that are usually referred to the local coordinate system defined by the copolar and cross-polar fields according to the third Ludwig definition [17] or its extension to elliptical polarization. Thus, the field pattern can by expressed as $\vec{F}_{n}(\theta, \phi)=F_{n}(\theta, \phi) \hat{p}(\theta, \phi)$ where $F_{n}(\theta, \phi)$ is the scalar complex pattern appearing in (6) and (7), and $\hat{p}(\theta, \phi)$ is a unit vector having the polarization and orientation information. It is important to point out that each antenna pattern appearing in (6) and (7) includes the effect of the other antenna with matched output. In other words, $F_{n_{i}}(\theta, \phi)$ is the normalized voltage pattern of the structure formed by both antennas, measured at port $i$ and assuming both ports connected to the reference impedance. The term $D_{i}$ is the maximum directivity associated to the radiation from port $i$. This means that $D_{i}\left|F_{n_{i}}(\theta, \phi)\right|^{2}$ is the ratio between the radiation intensity produced by the structure transmitting from port $i$ to the total radiated power divided by $(4 \pi)$, computed in the direction $(\theta, \phi)$.

The brightness temperature $T_{\mathrm{B}}(\theta, \phi)$ appearing in (6) should be computed from the component of the electric field parallel to $\hat{p}(\theta, \phi)$ (i.e., the scalar product of the field with the unit vector). For an unpolarized radiation, such as atmospheric emission, this effect is irrelevant, but for off-nadir ground emission, a rotation matrix [18] must be applied at each direction $(\theta, \phi)$ in order to refer the measurements to the standard horizontal and vertical polarization basis. In this case, $T_{\mathrm{B}}(\theta, \phi)$ in (6) is in fact a combination of the Stokes parameters of the source. If the antenna beam is narrow this effect is negligible and the power collected by the antenna turns out to be directly related to the vertical and horizontal brightness temperature of the ground spot. An interferometric aperture synthesis radiometer, however, uses wide-beam antennas.

Similarly, the brightness temperature $T_{\mathrm{B}}(\theta, \phi)$ appearing in (7) should be computed as the cross correlation between the two field components parallel to each one of the two antenna polarization unit vectors. If both have the same direction, then this is equivalent to the definition in (6), so both equations become consistent and the same comments apply. On the other hand, if both antennas have orthogonal polarization, $T_{\mathrm{B}}$ is a complex function having as real and imaginary parts the rotated third and fourth stokes parameters of the source. This is the basis of the polarimetric mode of MIRAS [19].

Substituting (5)-(7) in (3) and (4)

$$
\begin{aligned}
& \overline{\left|b_{i}\right|^{2}}=\mathcal{K}\left(\left|S_{i i}\right|^{2} T_{i}+\left|S_{i j}\right|^{2} T_{j}+T_{a_{i}}\right) \\
& \overline{b_{i} b_{j}^{*}}=\mathcal{K}\left(S_{i i} S_{j i}^{*} T_{i}+S_{i j} S_{j j}^{*} T_{j}+\mathcal{V}_{i j}\right)
\end{aligned}
$$

which show that the power and cross-power spectral density of the output waves are function not only of the antenna temperature and the visibility, but also of the noise generated by the receivers, characterized by their temperatures. If one assumes 
that the antennas are well matched and their coupling is negligible $\left(S_{i j}=0 \forall i, j\right)$, then $T_{a_{i}}$ and $\mathcal{V}_{i j}$ become only related to the power and cross-power density of the output waves. To our knowledge, in all the preceding analysis this assumption was always implicit. However, as it will be shown in the next section, these S-parameters cannot be made equal to zero, especially for small antenna spacing. Moreover, since the antenna temperature is usually large, the approximation has low impact in (8), but it is important in (9) because of the usually low value of the visibility. It can be argued that the visibility is larger for small antenna spacing, but in this case the antenna coupling is also larger.

A special case of interest is that in which both receivers are at the same temperature $\left(T_{i}=T_{j}=T_{r}\right)$. Then, (8) and (9) become

$$
\begin{aligned}
& \overline{\left|b_{i}\right|^{2}}=\mathcal{K}\left[\left(\left|S_{i i}\right|^{2}+\left|S_{i j}\right|^{2}\right) T_{r}+T_{a_{i}}\right] \\
& \overline{b_{i} b_{j}^{*}}=\mathcal{K}\left[\left(S_{i i} S_{j i}^{*}+S_{i j} S_{j j}^{*}\right) T_{r}+\mathcal{V}_{i j}\right] .
\end{aligned}
$$

In the following subsections, a general relation between S-parameters and antenna patterns is obtained, which will be used to write the above equations in a more compact form.

\section{A. Thermodynamic Equilibrium}

The above equations must also hold if the thermal source consists of a microwave absorber completely surrounding the antennas (anechoic chamber) at a constant temperature $T_{0}$, and the receivers are also at the same temperature (thermodynamic equilibrium). In this situation, application of the Bosma theorem [14], [20], shows that, independent of the antenna design

$$
\overline{\left|b_{i}\right|^{2}}=\mathcal{K} T_{0} \quad \overline{b_{i} b_{j}^{*}}=0 .
$$

Introducing this known result in (10) and (11), he following relations are found while in thermodynamic equilibrium:

$$
\begin{aligned}
& T_{a_{i}}=T_{0}\left(1-\left|S_{i i}\right|^{2}-\left|S_{i j}\right|^{2}\right) \\
& \mathcal{V}_{i j}=-T_{0}\left(S_{i i} S_{j i}^{*}+S_{i j} S_{j j}^{*}\right)
\end{aligned}
$$

which could also have been directly obtained by application of the Bosma relation $\overline{\mathbf{b}_{\mathbf{s}} \mathbf{b}_{\mathbf{s}}^{\dagger}}=\mathcal{K} \mathbf{T}_{\mathbf{0}}\left(\mathbf{I}-\mathbf{S S}^{\dagger}\right)$ valid for any linear passive two-port at constant temperature [14], [20].

Now, using (6) and (7) with $T_{\mathrm{B}}=T_{0}$ for the left-hand side terms of (13) and (14), it follows that the antenna patterns must satisfy the following relations:

$$
\frac{D_{i}}{4 \pi} \iint_{4 \pi}\left|F_{n_{i}}(\theta, \phi)\right|^{2} d \Omega=\left(1-\left|S_{i i}\right|^{2}-\left|S_{i j}\right|^{2}\right)
$$

$\frac{\sqrt{D_{i} D_{j}}}{4 \pi} \iint_{4 \pi} F_{n_{i}}(\theta, \phi) F_{n_{j}}^{*}(\theta, \phi) e^{j k \Delta r} d \Omega=-\left(S_{i i} S_{j i}^{*}+S_{i j} S_{j j}^{*}\right)$.
It should be stressed that this is a general result, since it has been obtained using only a power balance approach. Moreover, it has been checked for several kind of antennas by numerical computation using the method of moments [21]. If the separation between both antennas is large, assuming properly designed antennas, all S-parameters are null, so the right-hand side of (15) becomes unity that of (16) vanishes.

\section{B. General Case}

The relations (15) and (16) can now be used to write the general equations (10) and (11) in a convenient form by grouping terms and using (6) and (7)

$$
\begin{aligned}
& \overline{\left|b_{i}\right|^{2}}=\mathcal{K}\left(T_{a_{i}}+T_{r} \mathcal{I}_{i}\right) \\
& \overline{b_{i} b_{j}^{*}}=\frac{\mathcal{K} \sqrt{D_{i} D_{j}}}{4 \pi} \iint_{4 \pi}\left(T_{\mathrm{B}}-T_{r}\right) F_{n_{i}} F_{n_{j}}^{*} e^{j k \Delta r} d \Omega
\end{aligned}
$$

where the explicit dependence on $(\theta, \phi)$ of the brightness temperature and of the antenna patterns has been omitted to simplify the notation. The term $\mathcal{I}_{i}$ in (17) is defined as

$$
\mathcal{I}_{i}=1-\frac{D_{i}}{4 \pi} \iint_{4 \pi}\left|F_{n_{i}}(\theta, \phi)\right|^{2} d \Omega
$$

and should always be much lower than unity and zero for distant antennas. These equations assume that the temperature $T_{r}$ of both receivers is the same. Obviously this is a simplification, but allows to show an important result. In general, the cross correlation of the total output waves (18) depends on the difference between the brightness temperature of the source and the receivers physical temperature, provided that the receivers have good input isolators.

This important result deserves further comments. If the antennas are sufficiently separated apart so that (16) equals zero, then there is no need to subtract $T_{r}$ to $T_{\mathrm{B}}$ in (18), since the weighted contribution of $T_{r}$ with the antenna pattern vanishes. In this case, the correlation of the receivers' outputs becomes directly proportional to the visibility, which can also be seen from (11). An example of this situation is the Very Large Array in New Mexico, intended to measure a distant star. For earth observation, the drawback of having the antennas too separated is that the alias-free field of view reduces drastically [8], so the instrument would only be useful for measuring sources of small extent. To get more insight in this matter, Fig. 2 shows the computation of the left-hand side of (16) using the measured antenna patterns of the units fabricated by EADS-Casa Espacio for MIRAS. They are dual linear polarization patch antennas having 10-dB directivity. The circles in the figure indicate the nominal antenna separation of the instrument, showing clearly that the impact of this effect may be not negligible and should be taken into account in the inversion process.

Experimental evidence of (18) is given in [12], which presents measurements of cross correlation between pairs of receivers at different positions inside an anechoic chamber. Using simply (7) with $T_{\mathrm{B}}$ constant, it follows that the cross correlation should 


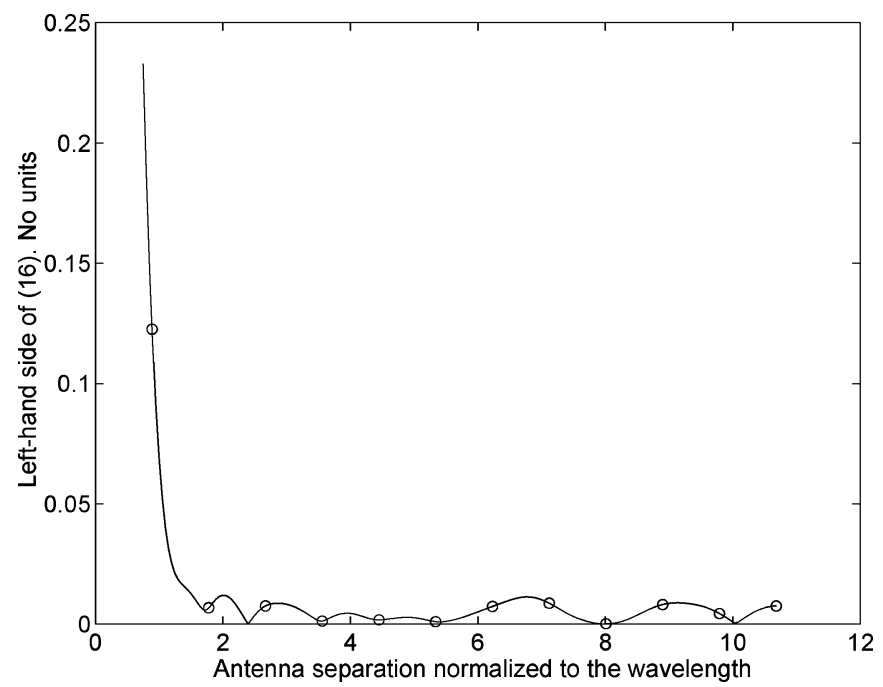

Fig. 2. Computation of (16) from actual measurements of MIRAS antennas. The circles show the antenna location in the instrument.

be equal to the left-hand side of (16), which from figure (2) is clearly nonzero for close antennas. However, the experiment showed always zero cross correlation, which is consistent with (18) if the anechoic chamber is at the same temperature as the receivers.

\section{Filtering AND Time-Domain CORRELATION}

In practice, the instrument performs measurements in timedomain using a correlator and an integrator after filtering the signals, as shown in Fig. 1. In the Appendix, it is shown that assuming infinite integration time, the mean power and cross correlation of the time-domain analytic signals at the output of the receivers are given by

$$
\begin{aligned}
\frac{1}{2}\left\langle\left|b_{0 i}(t)\right|^{2}\right\rangle & =\int_{0}^{\infty} \overline{\left|b_{i}(f)\right|^{2}}\left|H_{i}(f)\right|^{2} d f \\
\frac{1}{2}\left\langle b_{0 i}(t) b_{0 j}^{*}(t)\right\rangle & =\int_{0}^{\infty} \overline{b_{i}(f) b_{j}^{*}(f)} H_{i}(f) H_{j}^{*}(f) d f
\end{aligned}
$$

where the frequency dependence of $b_{i}$ and $b_{j}$ has been explicitly shown, and $H_{i}(f)$ is the complex frequency-domain transfer function of receiver $i$. Introducing (17) and (18) into these equations, interchanging the order of integration, and adding the term due to the noise generated by the receivers that flows toward the correlator, the following time-domain formulas are obtained:

$$
\begin{aligned}
\frac{1}{2}\left\langle\left|b_{0 i}(t)\right|^{2}\right\rangle= & \mathcal{K} B_{i} G_{i}\left(T_{a_{i}}+T_{r} \mathcal{I}_{i}+T_{R_{i}}\right) \\
\frac{1}{2}\left\langle b_{0 i}(t) b_{0 j}^{*}(t)\right\rangle= & \frac{\mathcal{K} \sqrt{D_{i} D_{j}} \sqrt{B_{i} B_{j}} \sqrt{G_{i} G_{j}}}{4 \pi} \\
& \cdot \iint_{4 \pi}\left(T_{\mathrm{B}}-T_{r}\right) F_{n_{i}} F_{n_{j}}^{*} \tilde{r}_{i j}\left(\frac{\Delta r}{c}\right) e^{j k_{0} \Delta r} d \Omega
\end{aligned}
$$

where $\mathcal{I}_{i}$ is defined in (19), $T_{R_{i}}$ is the noise equivalent temperature of receiver $i, k_{0}$ is the wavenumber at the center frequency $f_{0}, G_{i}$ is the power gain of receiver $i$, and the noise bandwidths $B_{i}$ and fringe washing function $\tilde{r}_{i j}(\tau)$ are defined as

$$
\begin{aligned}
B_{i} & =\frac{1}{G_{i}} \int_{0}^{\infty}\left|H_{i}(f)\right|^{2} d f \\
\tilde{r}_{i j}(\tau) & =\frac{e^{-j 2 \pi f_{0} \tau}}{\sqrt{B_{i} B_{j}} \sqrt{G_{i} G_{j}}} \int_{0}^{\infty} H_{i}(f) H_{j}^{*}(f) e^{j 2 \pi f \tau} d f .
\end{aligned}
$$

It should be noted that if the input isolators of the receivers have poor isolation, then (23) must include an additional term to take into account the partial correlation between the noise waves generated by the receivers, as discussed in Section II.

From (23), it is useful to redefine the visibility in terms of the actually measured time-domain cross correlation

$$
V_{i j}=\frac{1}{\mathcal{K} \sqrt{B_{i} B_{j}} \sqrt{G_{i} G_{j}}} \frac{1}{2}\left\langle b_{0 i}(t) b_{0 j}^{*}(t)\right\rangle .
$$

The final equation for this visibility comes from (23) after changing variables from angular coordinates to director cosines $(\xi=\sin \theta \cos \phi, \eta=\sin \theta \sin \phi)$, writing the differential solid angle as a function of these variables

$$
d \Omega=\sin \theta d \theta d \phi=\frac{d \xi d \eta}{\sqrt{1-\xi^{2}-\eta^{2}}}
$$

and assuming parallax approximation for the computation of $\Delta r$, considering both antennas in the $X-Y$ plane

$$
\Delta r=r_{2}-r_{1} \approx-(\xi \Delta x+\eta \Delta y)
$$

With these modifications, this newly defined visibility function can be written as

$$
\begin{array}{r}
V_{i j}(u, v)=\iint_{\xi^{2}+\eta^{2} \leq 1} T_{i j}^{\prime}(\xi, \eta) \tilde{r}_{i j}\left(-\frac{u \xi+v \eta}{f_{0}}\right) \\
\cdot e^{-j 2 \pi(u \xi+v \eta)} d \xi d \eta
\end{array}
$$

where

$$
T_{i j}^{\prime}(\xi, \eta)=\frac{\sqrt{D_{i} D_{j}}}{4 \pi} \frac{T_{\mathrm{B}}(\xi, \eta)-T_{r}}{\sqrt{1-\xi^{2}-\eta^{2}}} F_{n_{i}}(\xi, \eta) F_{n_{j}}^{*}(\xi, \eta)
$$

and $u$ and $v$ are the projections over the $X-Y$ axes of the vector defined between the two phase centers of the antennas normalized to the wavelength $u=\left(x_{2}-x_{1}\right) / \lambda_{0}, v=\left(y_{2}-y_{1}\right) / \lambda_{0}$.

Finally, using (22) and (26), the normalized visibility is given by

$$
\mu_{i j}(u, v)=\frac{\left\langle b_{0 i}(t) b_{0 j}(t)^{*}\right\rangle}{\sqrt{\left\langle\left|b_{0 i}(t)\right|^{2}\right\rangle\left\langle\left|b_{0 j}(t)\right|^{2}\right\rangle}}=\frac{V_{i j}(u, v)}{\sqrt{T_{\text {sys }_{i}} T_{\text {sys }_{j}}}}
$$


where $V_{i j}(u, v)$ is the visibility (29), and $T_{\text {sys }_{i}}$ is the system temperature of receiver $i$

$$
T_{\mathrm{sys}_{i}}=T_{a_{i}}+T_{r} \mathcal{I}_{i}+T_{R_{i}}
$$

in which for most practical situations the approximation $T_{r} \mathcal{I}_{i} \approx$ 0 can be applied.

\section{CONCLUSION}

A formal derivation of the fundamental equation of interferometric radiometry is presented. This is a Fourier-like integral transformation between the brightness temperature map of the source $T_{\mathrm{B}}(\xi, \eta)$ and the visibility function $V_{i j}(u, v)$. The main difference between the present result and the usual equation found in the literature [22] is that the transformed function is not solely the brightness temperature, but instead the difference between this and the receivers physical temperature. This is due to the combined effect of mutual coupling and matching of the antennas and the thermal noise generated by the receivers. Another difference is that, due to the wide beamwidth of the individual antennas, the brightness temperature map does not correspond to a single polarization. Instead, at each direction of space, the brightness temperature follows the orientation of the polarization vector of the antennas at that direction.

\section{APPENDIX \\ DETAILED DERIVATION OF (20) AND (21)}

The power and cross-power spectral densities of the output signals $b_{0 i}(f)$ in Fig. 1 can be written as a function of those of the input signals $b_{i}(f)$ by using the following standard relations (e.g., see [23, eqs. (10-38) and (10-46)])

$$
\begin{aligned}
\overline{\left|b_{0 i}(f)\right|^{2}} & =\frac{1}{2} \overline{\left|b_{i}(f)\right|^{2}}\left|H_{i}(f)\right|^{2} \\
\overline{b_{0 i}(f) b_{0 j}^{*}(f)} & =\frac{1}{2} \overline{b_{i}(f) b_{j}^{*}(f)} H_{i}(f) H_{j}^{*}(f)
\end{aligned}
$$

where $H_{i}(f)$ is the frequency-domain transfer function of receiver $i$. The terms $1 / 2$ are included for consistency with the definition of $\left|b_{i}(f)\right|^{2}$ given in Section II as the total power in a $1-\mathrm{Hz}$ bandwidth. The correct definition of power spectral density must include this factor to take into account positive and negative frequencies. Now, according to the equation without number after (10-57) in [23], the spectral densities of the corresponding analytic signals are

$$
\begin{aligned}
\overline{\left|\hat{b}_{0 i}(f)\right|^{2}} & = \begin{cases}4 \overline{\left|b_{0 i}(f)\right|^{2}}, & \text { for } f>0 \\
0, & \text { for } f<0\end{cases} \\
\overline{\hat{b}_{0 i}(f) \hat{b}_{0 j}^{*}(f)} & = \begin{cases}4 \overline{b_{0 i}(f) b_{0 j}^{*}(f),} & \text { for } f>0 \\
0, & \text { for } f<0\end{cases}
\end{aligned}
$$

where the hat is used to denote analytic signal. Using now [23, eqs. (10-16) and (10-20)], the auto- and cross-correlation functions of the time-domain analytic signals at the origin $(\tau=0)$ are found by integrating over all frequencies the corresponding spectral densities

$$
\begin{aligned}
\left\langle\left|\hat{b}_{0 i}(t)\right|^{2}\right\rangle & =\int_{-\infty}^{\infty} \overline{\left|\hat{b}_{0 i}(f)\right|^{2}} d f=4 \int_{0}^{\infty} \overline{\left|b_{0 i}(f)\right|^{2}} d f \\
\left\langle\hat{b}_{0 i}(t) \hat{b}_{0 j}^{*}(t)\right\rangle & =\int_{-\infty}^{\infty} \overline{\hat{b}_{0 i}(f) \hat{b}_{0 j}^{*}(f)} d f \\
& =4 \int_{0}^{\infty} \overline{b_{0 i}(f) b_{0 j}^{*}(f)} d f .
\end{aligned}
$$

Now, by inserting (33) and (34) into these, (20) and (21) are readily obtained except that, in order to simplify the notation, the hat to denote analytic signal has been omitted.

\section{REFERENCES}

[1] C. S. Ruf, C. T. Swift, A. B. Tanner, and D. M. Le Vine, "Interferometric synthetic aperture microwave radiometry for the remote sensing of the Earth," IEEE Trans. Geosci. Remote Sensing, vol. 26, pp. 597-611, Sept. 1988.

[2] D. M. Le Vine, C. T. Swift, and M. Haken, "Development of the synthetic aperture microwave radiometer, ESTAR," IEEE Trans. Geosci. Remote Sensing, vol. 39, pp. 199-202, Jan. 2001.

[3] M. Martín-Neira and J. M. Goutoule, "A two-dimensional aperture-synthesis radiometer for soil moisture and ocean salinity observations," ESA Bull., no. 92, pp. 95-104, Nov. 1997.

[4] P. Silvestrin, M. Berger, Y. Kerr, and J. Font, "ESA's second Earth Explorer Opportunity mission: The Soil Moisture and Ocean Salinity mission-SMOS," IEEE Geosci. Remote Sensing Newslett., no. 118, pp. 11-14, Mar. 2001

[5] A. Camps, "Application of interferometric radiometry to earth observation," Ph.D. dissertation, Univ. Politècnica de Catalunya, Catalunya, Spain, Nov. 1996.

[6] J. Bará, A. Camps, F. Torres, and I. Corbella, "Angular resolution of twodimensional hexagonally sampled interferometric radiometers," Radio Sci., vol. 33, no. 5, pp. 1459-1473, Sept.-Oct. 1998.

[7] F. Torres, A. Camps, J. Bará, I. Corbella, and R. Ferrero, "On-board phase and modulus calibration of large aperture synthesis radiometers: Study applied to MIRAS," IEEE Trans. Geosci. Remote Sensing, vol. 34, pp. 1000-1009, July 1996.

[8] A. Camps, J. Bará, I. Corbella, and F. Torres, "The processing of hexagonally sampled signals with standard rectangular techniques: Application to 2D large aperture synthesis interferometric radiometers," IEEE Trans. Geosci. Remote Sensing, vol. 35, pp. 183-190, Jan. 1997.

[9] K. Rautiainen, R. Butora, T. Auer, N. Mononen, J. Salminen, S. Tauriainen, M. Hallikainen, J. Uusitalo, and P. Jukkala, "The Helsinki University of Technology/Ylinen electronics airborne L-band interferometric radiometer," in Proc. IGARSS, vol. 7, 2000, pp. 2978-2980.

[10] D. M. Le Vine, K. Carver, C. Swift, M. Foodberlet, I. Popstefanija, and J. Mead, "Development of a two dimensional synthetic aperture radiometer at L-band," in Proc. IGARSS, vol. 7, 2000, pp. 2994-2996.

[11] D. M. Le Vine, "Synthetic aperture radiometer systems," IEEE Trans. Microwave Theory Tech., vol. 47, pp. 2228-2236, Dec. 1999.

[12] N. Duffo, I. Corbella, M. Vall-llossera, A. Camps, M. Zapata, F. Torres, J. Benito, and J. Capdevila, "MIRAS imaging validation," in Proc. IGARSS, vol. 2, July, 21-25 2003, pp. 1226-1228.

[13] R. Hecken, "Analysis of linear noisy two-ports using scattering waves," IEEE Trans. Microwave Theory Tech., vol. MTT-29, pp. 997-1004, Oct. 1981.

[14] S. W. Wedge and D. B. Rutledge, "Noise waves and passive linear multiports," IEEE Microwave Guided Wave Lett., vol. 1, pp. 117-119, May 1991.

[15] — "Wave techniques for noise modeling and measurement," IEEE Trans. Microwave Theory Tech., vol. 40, pp. 2004-2012, Nov. 1992.

[16] A. R. Thomson, J. M. Moran, J. George, and W. Swenson, Interferometry and Synthesis in Radio Astronomy. New York: Wiley, 1986.

[17] A. C. Ludwig, "The definition of cross polarization," IEEE Trans. Antennas Propagat., vol. AP-21, pp. 116-119, Jan. 1973. 
[18] A. J. Gasiewski and D. B. Kunkee, "Calibration and applications of polarization correlating radiometers," IEEE Trans. Microwave Theory Tech., vol. 41, pp. 767-773, May 1993.

[19] M. Martín-Neira, S. Ribó, and A. J. Martín-Polegre, "Polarimetric mode of MIRAS," IEEE Trans. Geosci. Remote Sensing, vol. 40, pp. 1755-1768, Aug. 2002.

[20] H. Bosma, "On the theory of linear noisy systems," Ph.D. dissertation, Technische Hogeschool Te Eindhoven, The Nederlands, 1967.

[21] S. Blanch, J. Romeu, and I. Corbella, "An exact representation of an antenna system diversity performance from its input parameter description," IEE Electron. Lett., vol. 39, pp. 705-706, May 2003.

[22] M. Martín-Neira, "Introduction to two-dimensional aperture synthesis microwave radiometry for earth observation: Polarimetric formulation of the visibility function," ESTEC, Eur. Space Agency, Noordwijk, The Netherlands, Tech. Rep. Internal Working Paper 2130, Oct. 2001.

[23] A. Papoulis, Probability, Random Variables and Stochastic Processes. New York: McGraw-Hill, 1965.

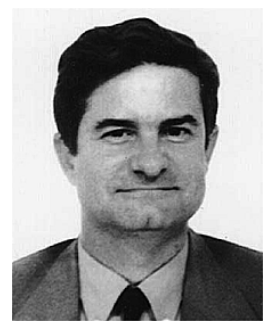

Ignasi Corbella (M'99) received the Telecommunications Engineering and Dr.Eng. degrees, both from Universitat Politècnica de Catalunya (UPC), Barcelona, Spain, in 1977 and 1983, respectively.

In 1976, he joined the School of Telecommunication Engineering in UPC as a Research Assistant in the Microwave Laboratory, where he worked on passive microwave integrated circuit design and characterization. During 1979, he worked at ThomsonCSF, Paris, France, on microwave oscillators design. In 1982, he became an Assistant Professor at UPC, an Associate Professor in 1986, and a Full Professor in 1993. He is currently teaching microwaves at the undergraduate level in UPC and has designed and taught graduate courses on nonlinear microwave circuits. During the school year 1998-1999, he worked at NOAA/Environmental Technology Laboratory, Boulder, CO, as a Guest Researcher, developing methods for radiometer calibration and data analysis. His research work in the Department of Signal Theory and Communications, UPC includes microwave airborne and satellite radiometry and microwave system design.

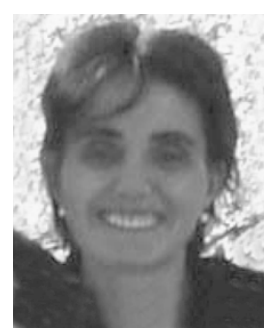

Núria Duffo (S'91-M'99) received the Telecommunication Engineer degree from the Polytechnic University of Catalonia (UPC), Barcelona, Spain, and the Doctor in Telecommunication Engineering from UPC, in 1990 and 1996, respectively.

Since 1997, she has been an Associate Professor at UPC. Her current research interests are numerical methods in electromagnetics, microwave radiometry, antenna analysis, and design.

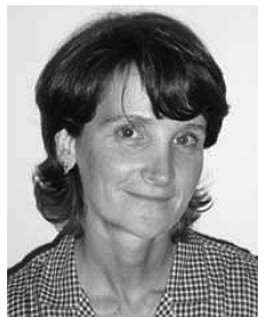

Mercè Vall-Ilossera (M'99) received the Senior Telecommunication Engineer and the Doctor Telecommunication Engineering degrees in 1990 and 1994, respectively, both from the Polytechnic University of Catalonia (UPC), Barcelona, Spain.

She has been lecturing and doing research at the Department of Signal Theory and Communications, UPC from 1990 until 1997 as an Assistant Professor and from 1997 until present as an Associate Professor. She spent a sabbatical year in Montreal with the scholarship of the "Programme Québécois de Bourses d'excellence" (1996-1997): "Stages de Formation postdoctorale au Québec pour jeunes diplômés étrangers.” Her research interests include numerical methods in electromagnetism, microwave radiometry, antenna analysis, and design. Currently, her research is mainly related to the study of numerical methods applied to the sea surface emissivity and their characterization at L-band and the MIRAS/SMOS project.

Dr. Vall-llossera, along with the other member of the radiometry group at UPC, was awarded the "Primer Premio Duran Farell de Investigación Tecnológica" in 2002, and the "Primer Premio Ciutat de Barcelona d'Investigació Tecnològica" in 2001.

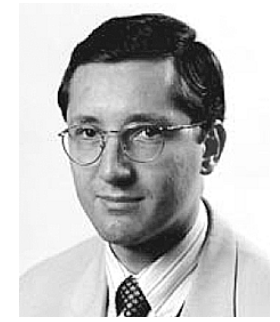

Adriano Camps (S'91-A'97-M'00-SM'02) was born in Barcelona, Spain, in 1969. He received the Telecommunications Engineering degree and the $\mathrm{Ph} . \mathrm{D}$. degree in telecommunications engineering in 1992 and 1996, respectively, both from the Polytechnic University of Catalonia (UPC), Barcelona, Spain.

From 1991 to 1992, he was with the ENS des Télécommunications de Bretagne, Bretagne, France, with an Erasmus Fellowship. In 1993, he joined the Electromagnetics and Photonics Engineering group, at the Department of Signal Theory and Communications, UPC, as an Assistant Professor, and since 1997 as an Associate Professor. In 1999, he was on sabbatical leave at the Microwave Remote Sensing Laboratory, University of Massachusetts, Amherst. His research interests are microwave remote sensing, with special emphasis in microwave radiometry by aperture synthesis techniques. He has performed numerous studies within the frame of European Space Agency SMOS Earth Explorer Mission. He is an Associate Editor of Radio Science.

Dr. Camps received the second national award of university studies in 1993, the INDRA award of the Spanish Association of Telecommunication Engineering to the best Ph.D. in 1997, the extraordinary Ph.D. award at the Universitat Politècnica de Catalunya in 1999, the First Duran Farell Award and the Ciudad de Barcelona Award, in 2000 and 2001, respectively, both for Technology Transfer; and in 2002, the Research Distinction of the Generalitat de Catalunya for contributions to microwave passive remote sensing. He was Chair of Cal '01. He is editor of the IEEE Geoscience and Remote Sensing Newsletter and President-Founder of the IEEE Geoscience and Remote Sensing Society Spain Chapter.

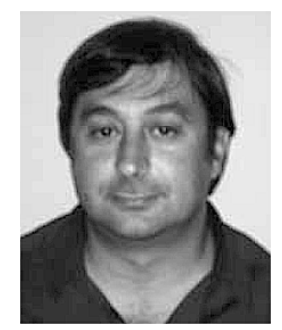

Francesc Torres (M'96) received the Ingeniero and Doctor Ingeniero degrees in telecommunication engineering from the Polytechnic University of Catalonia (UPC), Barcelona, Spain, in 1988 and 1992, respectively

In 1988-1989, he was a Research Assistant in the RF System Division, European Space Agency, Noordwijk, The Netherlands, devoted to microwave device testing and characterization. In 1989, he joined the Antenna-Microwave-Radar group, UPC, where he is currently an Associate Professor. His main research interests are focused on the design and testing of microwave systems and subsystems. He is currently engaged in research on interferometric radiometers devoted to earth observation. 